\title{
Boosting Relevance Model Performance with Query Term Dependence
}

\author{
Koji Eguchi \\ National Institute of Informatics \\ Tokyo 101-8430, Japan \\ eguchi@nii.ac.jp
}

\author{
W. Bruce Croft \\ Department of Computer Science \\ University of Massachusetts \\ Amherst, MA 01003, USA \\ croft@cs.umass.edu
}

Categories and Subject Descriptors: H.3.3 [Information Storage and Retrieval]: Information Search and Retrieval - Query formulation

General Terms: Performance, Experimentation

Keywords: information retrieval, term dependence, language models

\section{INTRODUCTION}

Phrase-based queries are known to perform effectively, especially against large-scale and noisy text data such as typically appear on the Web $[9,7,2]$. These methods can capture term dependencies that appear in a query. Meanwhile, a relevance model is a probability distribution over terms that is estimated from the set of relevant documents to address important notions of synonymy and polysemy [5]. The relevance model is usually estimated with unigrams from the results of pseudo-relevance feedback, i.e., from the topranked documents retrieved in response to a query. The combination of phrase-based query structuring and query expansion via the relevance model is promising, because each model has its own advantages: phrase-based query structuring attempts to address problems with bag-of-words representations and the relevance model attempts to address problems with simple word matching. In this paper, we investigate how query structuring with term dependence can improve the performance of query expansion via a relevance model. Some preliminary results can be found in the investigations of our research group $[8,1]$. In this paper, we attempt to examine the above point thoroughly.

\section{TERM DEPENDENCE MODELS}

Metzler and Croft developed a general, formal framework for modeling term dependencies via Markov random fields [7], and showed that the model is very effective in a variety of retrieval situations using the Indri platform [6]. Markov random fields (MRFs) are commonly used to model joint distributions succinctly. In [7], the joint distribution $P_{\Lambda}(Q, D)$ over queries $Q$ and documents $D$, parameterized by $\Lambda$, was modeled using MRFs, and for ranking purposes the posterior $P_{\Lambda}(D \mid Q)$ was derived by the following ranking function, assuming a graph $G$ that consists of a document node and query term nodes: $P_{\Lambda}(D \mid Q) \stackrel{\text { rank }}{=} \sum_{c \in C(G)} \lambda_{c} f(c)$, where $Q=t_{1} \ldots t_{n}, C(G)$ is the set of cliques in an MRF graph $G, f(c)$ is some real-valued feature function over clique values, and $\lambda_{c}$ is the weight given to that particular feature function. Three variants of the MRF model were assumed: (1)full-independence variant assumes that query terms are independent of each other; (2) sequential dependence variant assumes dependence between neighboring query terms; and

Copyright is held by the author/owner(s).

CIKM'06, November 5-11, 2006, Arlington, Virginia, USA.

ACM 1-59593-433-2/06/0011.
(3) full-dependence variant assumes that all query terms are in some way dependent on each other. To express these assumptions, the following ranking function was derived:

$P_{\Lambda}(D \mid Q) \stackrel{\mathrm{rank}}{=} \sum_{c \in T} \lambda_{T} f_{T}(c)+\sum_{c \in O} \lambda_{O} f_{O}(c)+\sum_{c \in O \cup U} \lambda_{U} f_{U}(c)$

where $T$ is defined as the set of 2-cliques involving a query term and a document $D, O$ is the set of cliques containing the document node and two or more query terms that appear contiguously within the query, and $U$ is the set of cliques containing the document node and two or more query terms appearing noncontiguously within the query.

We further developed a two-stage term dependence model that captures long- and short-range dependencies differently [2], which can be considered to be generalized from Metzler and Croft's model. We assumed that: (i)global dependencies between query components are explicitly separated in a query; and (ii)local dependencies occur between constituents within a compound word when the compound word appears in a query component. The phrase-based query structuring used in this paper is based on this model. To achieve the model mentioned above, we extended Eq. (1), as follows [2]:

$$
\begin{aligned}
P_{\Lambda}(D \mid Q) \stackrel{\mathrm{rank}}{=} & \sum_{c_{q} \in T(Q)} \lambda_{T} f_{T}\left(c_{q}\right)+\sum_{c_{q} \in O(Q)} \lambda_{O} f_{O}\left(c_{q}\right) \\
+ & \sum_{c_{q} \in O(Q) \cup U(Q)} \lambda_{U} f_{U}\left(c_{q}\right) \\
f_{T}\left(c_{q}\right) & =f_{T}^{\prime}\left(\sum_{q_{k} \in c_{q}} \sum_{c_{t} \in T\left(q_{k}\right)} \mu_{T} g_{T}\left(c_{t}\right)\right) \\
f_{O}\left(c_{q}\right) & =f_{O}^{\prime}\left(\sum_{q_{k} \in c_{q}} \sum_{c_{t} \in O\left(q_{k}\right)} \mu_{O} g_{O}\left(c_{t}\right)\right) \\
f_{U}\left(c_{q}\right) & =f_{U}^{\prime}\left(\sum_{q_{k} \in c_{q}} \sum_{c_{t} \in O\left(q_{k}\right) \cup U\left(q_{k}\right)} \mu_{U} g_{U}\left(c_{t}\right)\right) .
\end{aligned}
$$

Here, $Q$ consists of query components $q_{1} \cdots q_{k} \cdots q_{m}$, and each query component consists of individual terms $t_{1} \cdots t_{n}$. $T(Q), O(Q)$ and $U(Q)$ can be defined in the same manner as in Eq. (1) with the query components consisting of a whole query, while $T\left(q_{k}\right), O\left(q_{k}\right)$ and $U\left(q_{k}\right)$ are defined with the individual terms consisting of a query component. The feature functions $f_{T}^{\prime}, f_{O}^{\prime}$ and $f_{U}^{\prime}$ and another feature functions $g_{T}$, $g_{O}$ and $g_{U}$ can be given in the same manner as $f_{T}, f_{O}$ and $f_{U}$ that were defined in Eq. (1), respectively. Hereafter, we assumed that the constraint $\lambda_{T}+\lambda_{O}+\lambda_{U}=1$ was imposed independently of the query, and we assumed $\mu_{T}, \mu_{O}$ and $\mu_{U}$ were determined by $1 / \#\left(c_{t}, c_{q}\right)$ for the functions $f_{T}^{\prime}, f_{O}^{\prime}$ and $f_{U}^{\prime}$, respectively, where $\#\left(c_{t}, c_{q}\right)$ represents the number of times the clique of terms $c_{t}$ was observed in the clique of query components $c_{q}$. According to Eq. (2), we defined $g l f d+$ model as an instance of the two-stage term dependence 
model, considering special features of Japanese [1,2]. The $g l f d+$ model expresses the dependencies between query components on the basis of the full dependence. It also expresses the dependencies between constituent words within a query component on the basis of the sequential dependence. The two-stage term dependence model should be reasonable for other languages, if query components can be specified in a query, for example "ozone hole, human body".

\section{RELEVANCE MODELS}

Lavrenko and Croft formulated relevance models that explicitly incorporated relevance into the language modeling [5] Following [8], we use the relevance models as a pseudorelevance feedback function in the framework of inference network-based retrieval models, as briefly described in the following. Given an initial query $Q_{\text {orig }}$, we retrieve a set of \# docs $f b$ documents and form a relevance model from them. We then form $Q_{r m}$ by wrapping the \#combine operator of Indri [6], around the most likely \#terms ${ }_{f b}$ terms from the relevance model that are not stopwords. Finally, an expanded query is formed that has the following form: \#weight $\left(\nu Q_{\text {orig }}(1.0-\nu) Q_{r m}\right)$, where \#weight indicates an Indri operator [6]. In this paper, we formulate $Q_{\text {orig }}$ using the two-stage term dependence model described in Section $\mathbf{2}$, instead of the usual full-independence $(f i)$ model.

\section{EXPERIMENTS}

We used a 100-gigabyte web document collection, $N W 100 G$ 01, which consisted of web documents gathered from the .jp domain and thus were mostly written in Japanese. The NW100G-01 was used in a series of NTCIR WEB task $[4,3$, 10]. In the experiments described in the following, we only used the terms specified in the title field of each topic statement. All the topics were written in Japanese, and so we performed morphological analysis using the MeCab tool ${ }^{1}$ to segment the title portion of the topic and to add POS tags. The definition of the title is different from the one used in a series of TREC, ${ }^{2}$ for instance, in the following ways: (i)the title field gives 1-3 components that were delimited by commas; and (ii)each of these components is supposed to indicate a certain concept, and so it sometimes consists of a single word, but it may also consist of a compound word [4].

For training, we experimented using the $g l f d+$ model with relevance model, as described in Section 3, over the relevance judgment data used in the NTCIR-3 WEB task [4], changing the weight $\nu, \#$ docs $_{f b}$ and \#terms ${ }_{f b} .{ }^{3}$ For testing, we used the relevance judgment data that were used in the NTCIR-5 WEB task [10]. For the relevance model, we used the top-ranked 5 and 10 documents $\left(\#\right.$ docs $\left._{f b}\right)$, and 5, 10 and 20 terms $\left(\#\right.$ terms $_{f b}$ ) for feedback. We used the optimized value of the weight $\nu$, which we obtained from training, corresponding to each pair of $\left(\#\right.$ docs $_{f b}$, \#terms $\left.{ }_{f b}\right)$ above. The results are shown in Table 1 . In this table, 'AvgPrec $a$ ' indicates the mean average precision over all the 35 topics. ' $\% \operatorname{chg}_{f}$ ' and ' $\%$ chg $_{t}$ ' were calculated on the bases of (i)each model without the relevance model (i.e., $f i$ alone or $g l f d+$ alone); and (ii)the $f i$ model alone, respectively. As shown in this table, the $g l f d+$ model alone worked $13 \%$ better than the $f i$ model alone, as also reported in [2]. The combination of the $g l f d+$ model and the relevance model achieves 8-12\% improvements over the $g l f d+$ model alone. Combining with the relevance model, the $g l f d+$ model worked 9$15 \%$ better than the $f i$ model under the same conditions of $\left(\#\right.$ docs $_{f b}, \#$ terms $\left._{f b}\right)$.

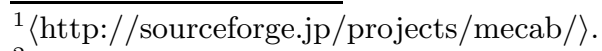

${ }^{2}\langle$ http://trec.nist.gov $/\rangle$.

${ }^{3}$ We also optimazed the parameters for the $g l f d+$ model using the NTCIR-3 WEB test collection, as described in [2].
}

Table 1: Evaluation results

\begin{tabular}{|c|c|c|c|c|}
\hline & $\nu$ & $\operatorname{AvgPrec}_{a}$ & $\%_{\text {chg }}$ & $\left(\% \operatorname{chg}_{t}\right)$ \\
\hline$f i$ alone & 1.0 & 0.1405 & +0.00 & $(+0.00$ \\
\hline$(05,05)$ & 0.9 & 0.1577 & $\left.+12.23^{*}\right]$ & $\left(+12.23^{* *}\right)$ \\
\hline$(05,10)$ & 0.9 & 0.1565 & $+11.40^{*}$ & $\left(+11.40^{* *}\right)$ \\
\hline$(05,20)$ & 0.9 & 0.1553 & $\left.+10.52^{*}\right]$ & $\left(+10.52^{* *}\right)$ \\
\hline$(10,05)$ & 0.9 & 0.1583 & $+12.66^{*}$ & $\left(+12.66^{* *}\right)$ \\
\hline$(10,10)$ & 0.9 & 0.1575 & $\left.+12.14^{*}\right]$ & $\left(+12.14^{* *}\right)$ \\
\hline$(10,20)$ & 0.9 & 0.1565 & $+11.39 *]$ & $(+11.39 * *)$ \\
\hline$g l f d+$ alone & 1.0 & 0.1588 & +0.00 & $\left(+13.02^{* *}\right)$ \\
\hline$(05,05)$ & 0.7 & 0.1730 & {$\left[+8.96^{*}\right]$} & $\left(+23.14^{* *}\right)$ \\
\hline$(05,10)$ & 0.7 & 0.1750 & $\left.+10.20^{*}\right]$ & $\left(+24.55^{* *}\right)$ \\
\hline$(05,20)$ & 0.5 & 0.1784 & +12.33 & $(+26.96 * *)$ \\
\hline$(10,05)$ & 0.7 & 0.1728 & $+8.80^{*}$ & $\left(+22.97^{* *}\right)$ \\
\hline$(10,10)$ & 0.7 & 0.1714 & {$[+7.95$} & $(+22.01 * *)$ \\
\hline$(10,20)$ & 0.5 & 0.1762 & +11.01 & $\left(+25.46^{* *}\right)$ \\
\hline \multicolumn{5}{|c|}{$\begin{array}{l}\text { In the left column, ' }(\cdot, \cdot) \text { ' indicates }(\# \text { docs } f b \text {, } \\
\# \text { terms } f b \text {. ' } * \text { and '**, indicate statistically sig- } \\
\text { nificant improvements over each model without the } \\
\text { relevance model (i.e., } f i \text { alone or glfd }+ \text { alone) and over } \\
\text { the } f i \text { model alone, respectively, where } p<0.05 \text { with } \\
\text { the two-sided Wilcoxon signed-rank test. }\end{array}$} \\
\hline
\end{tabular}

\section{CONCLUSIONS}

In this paper, we investigated how query structuring with term dependence could improve the performance of query expansion via a relevance model. We used the two-stage term dependence model to structure queries. We demonstrated through a series of experiments that the combination of the term dependence model and relevance model was more effective than either the term dependence model or the relevance model alone. Using the two-stage term dependence model with the relevance model, we achieved a significant $22-27 \%$ gain from the term independence model without using the relevance model.

Acknowledgments: We thank Donald Metzler for valuable discussions and comments. This work was supported in part by the Overseas Research Scholars Program and the Grants-inAid for Scientific Research (\#17680011 and \#18650057) from the Ministry of Education, Culture, Sports, Science and Technology, Japan, in part by the Telecommunications Advancement Foundation, Japan, and in part by the Center for Intelligent Information Retrieval. Any opinions, findings and conclusions or recommendations expressed in this material are those of the author(s) and do not necessarily reflect those of the sponsor.

\section{REFERENCES}

[1] K. Eguchi. NTCIR-5 query expansion experiments using term dependence models. In Proc. 5th NTCIR Workshop, Tokyo, Japan, 2005.

[2] K. Eguchi and W. B. Croft. Query structuring with two-stage term dependence in the Japanese language. In Information Retrieval Technology: Third Asia Information Retrieval Symposium, Singapore, LNCS 4182, pages 522-529. Springer, 2006.

[3] K. Eguchi, K. Oyama, A. Aizawa, and H. Ishikawa. Overview of the Informational Retrieval Task at NTCIR-4 WEB. In Proc. 4th NTCIR Workshop, Tokyo, Japan, 2004.

[4] K. Eguchi, K. Oyama, E. Ishida, N. Kando, and K. Kuriyama. Overview of the Web Retrieval Task at the Third NTCIR Workshop. In Proc. 3rd NTCIR Workshop, Tokyo, Japan, 2003.

[5] V. Lavrenko and W. B. Croft. Relevance based language models. In Proc. ACM SIGIR 2001, pages 120-127, New Orleans, Louisiana, USA, 2001.

[6] D. Metzler and W. B. Croft. Combining the language model and inference network approaches to retrieval. Information Processing and Management, 40(5):735-750, 2004.

[7] D. Metzler and W. B. Croft. A markov random field model for term dependencies. In Proc. ACM SIGIR 2005, pages 472-479, Salvador, Brazil, 2005.

[8] D. Metzler, T. Strohman, Y. Zhou, and W. B. Croft. Indri at TREC 2005: Terabyte Track. In Proc. TREC 2005, 2005.

[9] G. Mishne and M. de Rijke. Boosting web retrieval through query operations. In Advances in Information Retrieval: 27th European Conference on IR Research, Santiago de Compostela, Spain, LNCS 3408, pages 502-516. Springer, 2005.

[10] M. Yoshioka. Overview of the NTCIR-5 WEB Query Expansion Task. In Proc. 5th NTCIR Workshop, Tokyo, Japan, 2005. 